\title{
Genistein activates endothelial nitric oxide synthase in broiler pulmonary arterial endothelial cells by an Akt-dependent mechanism
}

\author{
Ying Yang ${ }^{1}$, Wei Nie ${ }^{1}$, Jianmin Yuan', \\ Bingkun Zhang ${ }^{1}$, Zhong Wang ${ }^{1}$, \\ Zhenlong $\mathrm{Wu}^{2,3}$ and Yuming Guo ${ }^{1,3}$ \\ ${ }^{1}$ State key Lab of Animal Nutrition \\ College of Animal Science and Technology \\ China Agricultural University (CAU) \\ Beijing 100193, China \\ ${ }^{2}$ College of Animal Science and Technology \\ CAU, Beijing 100193, China \\ ${ }^{3}$ Corresponding author: Tel, 86-10-62732712; \\ Fax, 86-10-62732712; E-mail, bio2046@ hotmail.com \\ DOI 10.3858/emm.2010.42.11.078
}

Accepted 30 September 2010

Available Online 6 October 2010

Abbreviations: Akt, protien kinase B; eNOS, endothelial nitric oxide synthase; ER, estrogen receptor; L-NAME, N-nitro-L-arginine methyl ester; PAECs, pulmonary arterial endothelial cells; PI3K, phosphatidylinositol 3-kinase

\begin{abstract}
Deregulation of endothelial nitric oxide synthase (eNOS) plays an important role in the development of multiple cardiovascular diseases. Our recent study demonstrated that genistein supplementation attenuates pulmonary arterial hypertension in broilers by restoration of endothelial function. In this study, we investigated the molecular mechanism by using broiler pulmonary arterial endothelial cells (PAECs). Our results showed that genistein stimulated a rapid phosphorylation of eNOS at Ser ${ }^{1179}$ which was associated with activation of eNOS/NO axis. Further study indicated that the activation of eNOS was not mediated through estrogen receptors or tyrosine kinase inhibition, but via a phosphatidylinositol 3-kinase (PI3K)/Akt-dependent signaling pathway, as the eNOS activity and related NO release were largely abolished by pharmacological inhibitors of PI3K or Akt. Thus, our findings revealed a critical function of Akt in mediating genistein-stimulated eNOS activity in PAECs, partially accounting for the beneficial effects of genistein on the development of cardiovascular diseases observed in animal models.
\end{abstract}

Keywords: endothelial cells; genistein; nitric oxide synthase type III; proto-oncogene proteins c-akt; pulmonary artery

\section{Introduction}

Cardiovascular diseases, such as coronary heart disease, hypertension and atherosclerosis are the leading causes of death among women in western countries. The observation that the incidence of cardiovascular diseases substantially increased in post-menopause women supports the viewpoint that the loss of protection attributed to estrogen deficiency (Grodstein et al., 1996). Further supportive evidence for the beneficial effects comes from the pre-clinical studies demonstrating that estrogen therapy reduces cardiovascular risk even though it increases breast cancer incidence after a prolonged treatment (Barrett-Connor and Bush, 1991; Stampfer and Colditz, 1991). The multiple benefits of estrogen have been reported to be mediated by several mechanism, including an enhanced expression of nitric oxide synthase (NOS), relaxation of pre-contracted vessels, inhibition of calcium entry, stimulation of the production of prostacyclin or an inhibitory effect on the development of myointimal hyperplasia (Farhat et al., 1996). Moreover, estrogen has also been shown to enhance endothelium-dependent vasodilation of coronary arteries in both postmenopausal women and primates (Farhat et al., 1996; Lissin and Cooke, 2000).

Given the demonstrated risks to conventional estrogen therapy, phytoestrogens, a class of natural occurring estrogen, have received increasing attention because of their potentially protective roles against several types of chronic diseases, includeing cardiovascular diseases, osteoporosis and hormone-related cancer (Cassidy and Griffin, 1999; Lissin and Cooke, 2000). Among phytoestrogens, genistein and daidzein, two major isoflavones in soybeans are the well-studied due to their wide distribution. In vitro and in vivo studies indicated that genistein supplementation has abilities to improve endothelial dysfunction in postmenopausal women, ovariectomized or chronically hypoxic rats (Squadrito et al., 2000; Karamsetty et al., 2001; Catania et al., 2002; Cuevas et al., 2003). Further 
A

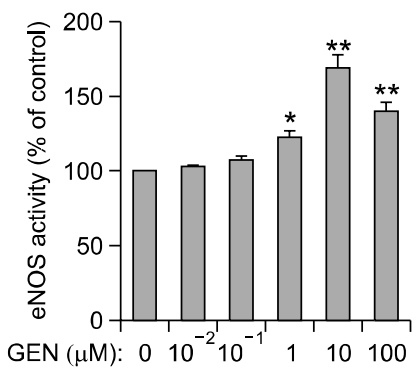

B

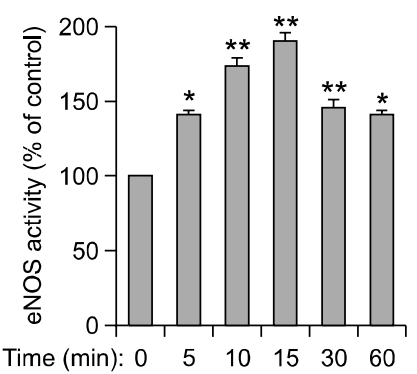

Figure 1. Genistein enhances eNOS activity in broiler pulmonary arterial endothelial cells (PAECs). (A) Serum-starved PAECs were treated with the indicated concentrations of genistein for $15 \mathrm{~min}$, eNOS activity was measured and represented as the mean \pm S.D. of three independent experiments. (B) Serum-starved cells were treated with $10 \mu \mathrm{M}$ genistein for the indicated time points and eNOS activity was measured. Each value represented the mean \pm S.D. of three independent experiments. ${ }^{*} P<$ 0.05 and ${ }^{*} P<0.01$ versus untreated control respectively.

studies shown that the beneficial effects of genistein were associated with the regulation on endothelial nitric oxide synthase (eNOS), because $\mathrm{N}$-nitro-L-arginine methyl ester (L-NAME), the inhibitor of eNOS, abolished the restoration of endothelial function in healthy postmenopausal women (Mishra et al., 2000; Colacurci et al., 2005) and rats (Vera et al., 2007). Upon activation, eNOS produces and release endothelial nitric oxide, a crucial vasoactive molecule in maintaining vascular homeostasis because of its potent activity to relax vascular arteries, prevent intra-vasal coagulation and platelet aggregation and antagonize the proliferation of smooth muscle cells ( $\mathrm{Li}$ and Forstermann, 2000). Deregulation of eNOS is an essential factor that correlated with the development

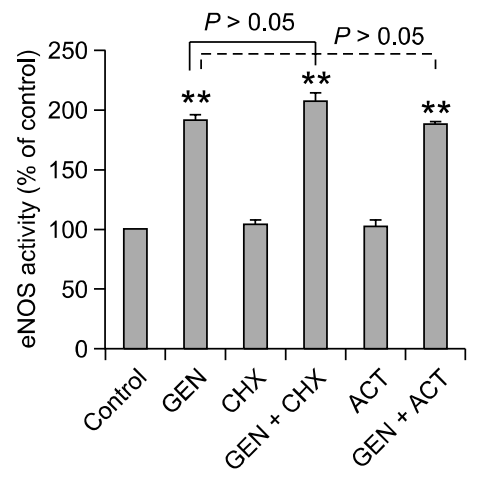

Figure 2. The rapid activation of eNOS by genistein is through a non-genomic mechanism. Serum-starved PAECs were pre-treated with protein synthesis inhibitor cycloheximide $(\mathrm{CHX}, 10 \mu \mathrm{M})$ or mRNA transcription inhibitor actinomycin D (ACT, $10 \mu \mathrm{M})$ for $30 \mathrm{~min}$, and then incubated with or without genistein (GEN, $10 \mu \mathrm{M}$ ) for additional $15 \mathrm{~min}$. eNOS activity was measured using the method described in materials and methods. Each value represented the mean \pm S.D. of three independent experiments, ${ }^{* *} P<0.01$ versus untreated control. of cardiovascular diseases and points to the possibility that genistein might directly or indirectly regulate eNOS activity in endothelial cells. However, the underlying mechanisms are not fully known.

Our recent study demonstrated that supplementation of genistein attenuated pulmonary hypertension by restoration of endothelial function in broilers (Yang et al., 2010). Therefore the aim of the present study was to investigate the effect of genistein on the activation of eNOS and the underlying mechanisms in broiler pulmonary arterial endothelial cells (PAECs).

\section{Results}

\section{Genistein enhances eNOS activity in broiler PAECs through a non-genomic mechanism involving phosphorylation of eNOS at Ser ${ }^{1179}$}

To determine the effect of genistein on the eNOS activity, cultured cells were treated with vehicle or indicated concentrations of genistien for $15 \mathrm{~min}$ (Figure $1 \mathrm{~A}$ ). In the range of $10^{-2}$ to $10^{2} \mu \mathrm{M}$ used in this experiment, genistein enhanced eNOS activity in a dose dependent manner, with genistein of 10 $\mu \mathrm{M}$ inducing maximal eNOS activation, which was observed as early as 5 min post-treatment and reach maximum after 15 min of incubation and then rapidly declined thereafter but was still significant higher than control after $1 \mathrm{~h}$ of genistein treatment (Figure 1B). The rapid activation of eNOS by genistein is by a non-genomic mechanism, because pre-treatment of the cells with cycloheximide, a protein synthesis inhibitor, or the mRNA transcription inhibitor actinomycin D had no significant effect on genistein-induced eNOS activity (Figure 2). eNOS has been reported to be 
A

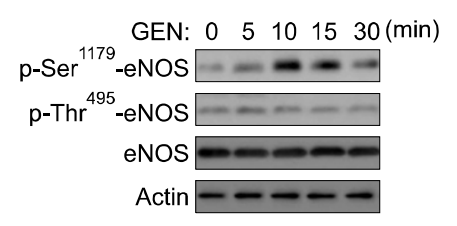

B

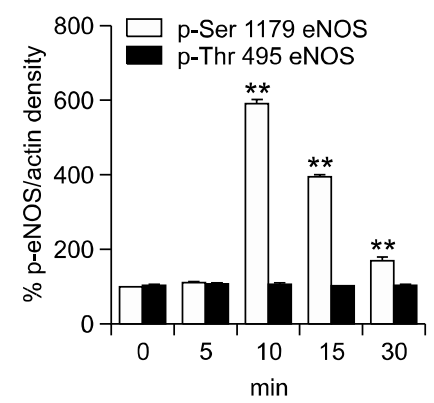

Figure 3. Genistein induced phosphorylation of eNOS at Ser ${ }^{1179}$ in PAECs. (A) Serum-starved PAECs were treated with genistein (GEN, $10 \mu \mathrm{M}$ ) for the indicated time points;western blot was performed to analyze the phosphorylation of eNOS at Ser ${ }^{1179}$ and $\mathrm{Thr}^{495}$, actin was used as a loading control. (B) Bar graph summarizing the western blot results by densitometry analysis. Values were means \pm S.D. of phospho-eNOS/ actin $(n=3)$ and expressed as percentage of control. ${ }^{* *} P<0.01$ versus untreated control.

activated by phosphorylation, we therefore investigated whether the rapid activation of eNOS induced by genistein is associated with phosphorylation. Western blot results showed that genistein treatment elicited a rapid increase of phosphorylation of eNOS at $\operatorname{Ser}^{1179}$, which was maximal at the concentration of $10 \mu \mathrm{M}$ (Figure 3); consistent with the enhanced eNOS activity induced by genistein (Figure 1A). In contrast, genistein treatment did not affect the phosphorylation of eNOS at Thr 495 (Figure 3), suggesting a critical role of phosphorylation of eNOS at $\mathrm{Ser}^{1179}$ in genistein-induced non-genomic eNOS activation.

\section{Genistein-induced activation of eNOS is not mediated by estrogen receptor (ER)}

It is well known that estrogen can regulate eNOS activity by a estrogen receptor mediated mechanism (MacRitchie et al., 1997). Genistein has weak estrogenic effect by binding to estrogen receptors due to the structural similarity to that of estrogen (Kuiper et al., 1997). We next evaluate whether ER was involved in genistein induced rapid activation of eNOS. Cells were incubated with genistein in the absence or presence of estrogen receptor antagonist ICI 182, 780 (100 $\mathrm{nM})$. As shown, ICI 182, 780 did not affect the eNOS activity (Figure 4A), nor the phosphorylation of eNOS at Ser ${ }^{1179}$ (Figures $4 \mathrm{~B}$ and $4 \mathrm{C}$ ), suggesting that genistein-induced activation of eNOS is not mediated by estrogen receptor in our system.

\section{Activation of eNOS in PAECs is independent of tyrosine kinase inhibition}

Beside the estrogenic effect, genistein is widely used as a tyrosine kinase inhibitor in various experimental systems; we next investigate whether the genistein-induced activation of eNOS is mediated by inhibition of tyrosine kinase in PAECs. Serum-starved endothelial cells were incubated with genistein or herbinycin $(10 \mu \mathrm{M})$, a specific inhibitor of tyrosine kinase, followed by tyrosine kinase activity assay. Genistein, at the concentrations used for the stimulation of eNOS activity in our experiment did not inhibit the tyrosine kinase activity (Figure 5). Our result is in agreement with the previous study showing that higher concentration of genistein $(100 \mu \mathrm{M})$ is required to inhibit tyrosine kinase activity (Liu et al., 2004). In contrast, herbimycin $\mathrm{A}$, a positive control used in our experiment, potently inhibit tyrosine kinase.

\section{Activation of eNOS by genistein is mediated by a PI3K/Akt-dependent mechanism}

eNOS can be phosphorylated at the Ser ${ }^{1179}$ and thus activated by several protein kinases, such as ERK (Bernier et al., 2000), Akt (Dimmeler et al., 1999; Fulton et al., 1999) or protein kinase A (PKA) (Boo et al., 2002) in response to various stimulus. To elucidate the potential kinase pathways responsible for genistein-induced eNOS phosphorylation, PAECs were pre-treated with vehicle or PKA inhibitor H89 $(5 \mu \mathrm{M})$, ERK inhibitor PD98059 (10 $\mu \mathrm{M})$, PI3K inhibitor LY294002 $(10 \mu \mathrm{M})$ or Akt inhibitor A443654 $(5 \mu \mathrm{M})$ for $30 \mathrm{~min}$ and then incubated with or without genistein $(10 \mu \mathrm{M})$ for another 15 min. As shown, H89 had no effect on 
A

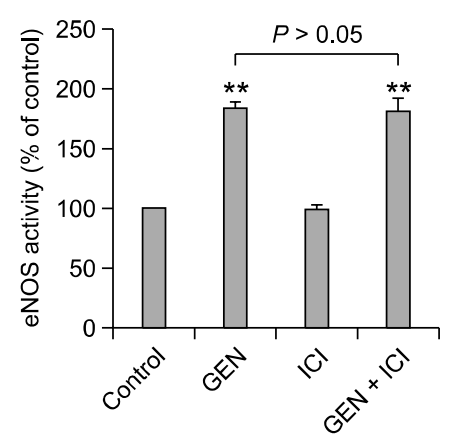

B

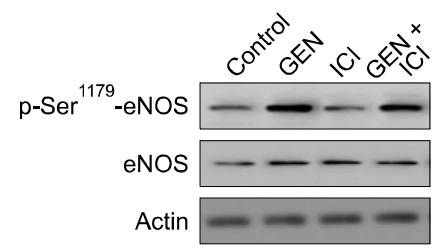

C

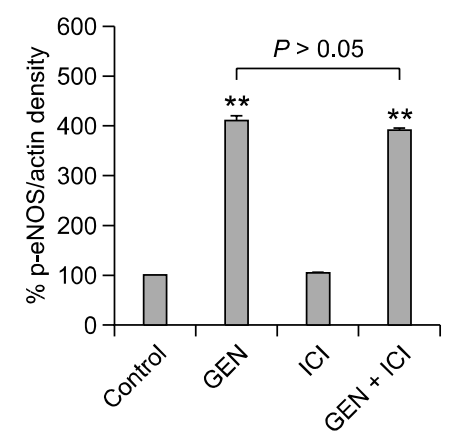

Figure 4. Genistein-induced activation of eNOS is not mediated by estrogen receptor (ER). (A) Serum-starved PAECs were treated with vehicle (control) or genistein (GEN, $10 \mu \mathrm{M})$ in the presence or absence of estrogen receptor antagonist ICl 182, $780(\mathrm{ICl}$, $100 \mathrm{nM}$ ) for $15 \mathrm{~min}$. eNOS activity was determined and represented as the mean \pm S.D. of three independent experiments. (B) Cells were treated as in (A) and harvested for western blot analysis using indicated antibodies. Actin was used as a loading control. (C) Bar graph summarizing the western blot results by densitometry analysis. Values were means \pm S.D. of phopho-eNOS/actin $(n=3)$ and expressed as percentage of control. ${ }^{* *} P<0.01$ versus untreated control.

genistein-induced eNOS activation (Figure 6A) and phosphorylation of eNOS at Ser ${ }^{1179}$ (Figures 6B and 6C). PD98059 did not affect genistein-induced eNOS activity, even though it partly abrogated

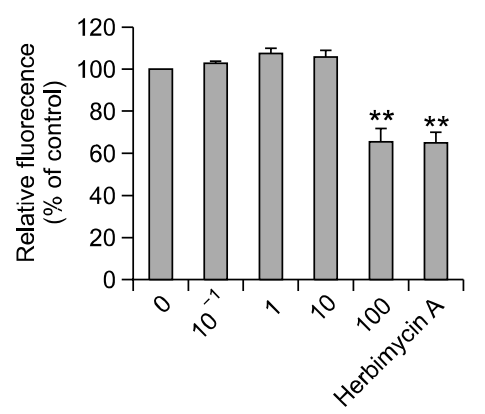

Figure 5. Genistein-induced activation of eNOS in PAECs is not mediated by tyrosine kinase inhibition. Serum-starved PAECs were pretreated with indicated concentrations of genistein, or herbimycin $A(10 \mu \mathrm{M})$ for 15 min, tyrosine kinase activity was determined and represented as the mean \pm S.D. of three independent experiments. ${ }^{* *} P<0.01$ versus un-treated control.
eNOS phosphorylation. By contrast, LY294002 and A443654 significantly abolished the phosphorylation of eNOS at Ser ${ }^{1179}$ and related eNOS activity, suggesting that genistein enhanced eNOS activity through a PI3K/Akt-dependent mechanism.

\section{Genistein stimulates NO release in pulmonary endothelial cells}

To further confirm the functional role of rapid activation of eNOS by genistein in PAECs, we determine the release of $\mathrm{NO}$ by the measuring of the sum concentration of $\mathrm{NO}_{2}^{-}$and $\mathrm{NO}_{3}^{-}$in culture supernatants using a fluoremetric assay. In consistence with the activation of eNOS, genistein (10 $\mu \mathrm{M})$ treatment significantly increased the NO release compared with that of control (Figure 7) which was completely blocked by pretreatment with L-NAME $(300 \mu \mathrm{M})$, an inhibitor of eNOS. In agreement with eNOS activity (Figure 6), genistein-stimulated NO release was significantly 


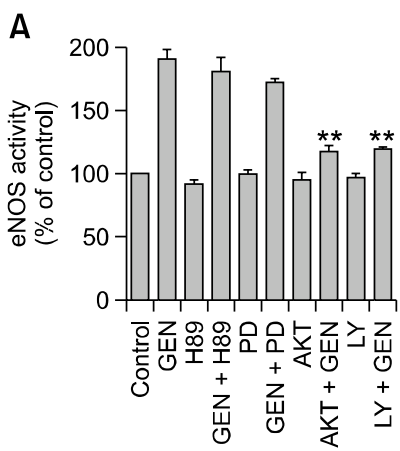

B
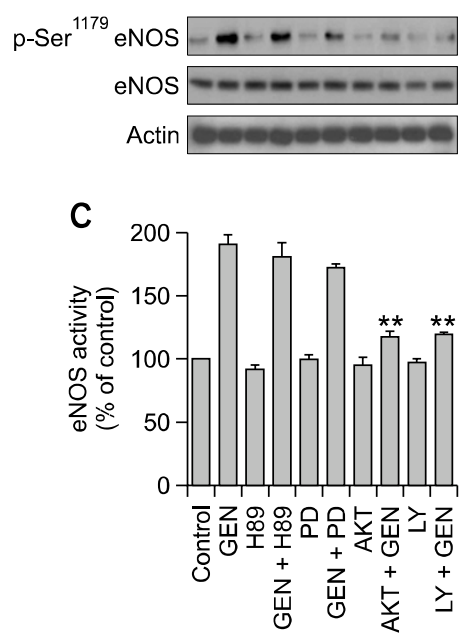

Figure 6. Activation of eNOS by genisein is mediated by a PI3K/Akt-dependent mechanism. (A) Serum-starved PAECs were pretreated with vehicle (control) or kinase inhibitors, H89 $(5 \mu \mathrm{M})$, PD98059 (PD, $10 \mu \mathrm{M})$, LY294002 (LY, $10 \mu \mathrm{M})$ or A443654 (A44, $5 \mu \mathrm{M})$ for 30 min and then incubated with or without genistein (GEN, $10 \mu \mathrm{M})$ for another $15 \mathrm{~min}$, eNOS activity were determined and represented as the mean \pm S.D. of three independent experiments. (B) Cells were treated as in (A) and harvested for western blot analysis using indicated antibodies. Actin was used as a loading control. (C) Bar graph summarizing the western blot results by densitometry analysis. Values were means \pm S.D. of phopho-eNOS 1179/actin $(n=3)$ and expressed as percentage of untreated control. ${ }^{* *} P<0.01$ versus genistein-stimulated eNOS activation.

blocked by PI3K inhibitor LY294002 $(10 \mu \mathrm{M})$ or Akt inhibitor A443654 (5 $\mu \mathrm{M})$, instead of PKA inhibitor H89 or ERK1/2 inhibitor PD98059.

\section{Discussion}

The phytoestrogen genistein has drawn increasing attention due to its potential healthy benefits in preventing cardiovascular diseases. However, the underlying mechanisms are still not well defined. In this study, we demonstrated that genistein stimulated a rapid activation of eNOS in a dose- and time-dependent manner. This effect was not mediated by a genomic mechanism since it occurred

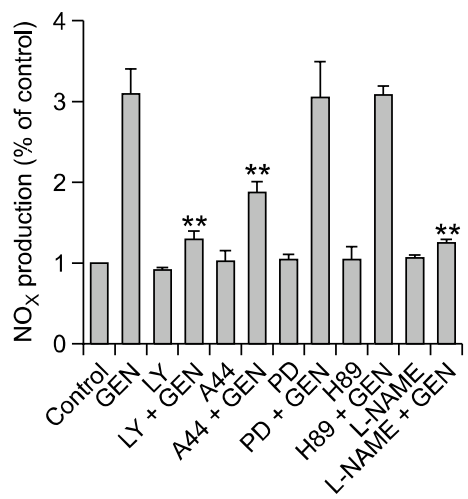

Figure 7. Genistein stimulates NO release in PAECs is PI3K/Akt-dependent. Serum-starved PAECs were treated with L-NAME $(300 \mu \mathrm{M})$, H89 $(5 \mu \mathrm{M})$, PD98059 (PD, $10 \mu \mathrm{M})$, A443654 (A44, $5 \mu \mathrm{M})$ or LY294002 $(\mathrm{LY}, 10 \mu \mathrm{M})$ for $30 \mathrm{~min}$ and then incubated with or without genistein (GEN, $10 \mu \mathrm{M}$ ) for another $15 \mathrm{~min}$, the production of NO was determined by measuring the concentration of $\mathrm{NO}_{2}^{-}$and $\mathrm{NO}_{3}^{-}\left(\mathrm{NO}_{\mathrm{x}}\right)$ in culture supernatants. Each value represented the mean \pm S.D. of three independent experiments. ${ }^{* *} P<0.01$ versus genistein-stimulated NO release.

rapidly and was not inhibited by cycloheximide or actinomycin D. Further study showed that genistein-induced eNOS activation through a PI3K/Aktdependent phosphorylation of eNOS at Ser ${ }^{1179}$ since the pretreatment of cells with $\mathrm{PI} 3 \mathrm{~K}$ inhibitor LY294002 or Akt inhibitor A443654 abrogated genistein stimulated eNOS activation (Figure 6) and related NO release (Figure 7), indicating an important role of PI3K/Akt in genistein-induced rapid activation of eNOS/NO in broiler PAECs.

Vascular endothelial cells play an important role in maintaining normal vascular function by producing several vasoconstrictors and vasodilators in response to multiple mechanical, physiological, and pharmacological stimuli (Schiffrin, 2001; Mawji and Marsden, 2003). NO produced by NOS in endothelial cells is a potent vasodilator in pulmonary vessels that maintains normal vascular tone and homeostasis through interactions with vasoconstrictor endothelin-1 (ET-1) (Lavallee et al., 2001). Impaired endothelial NO production contributes to the increased vascular resistance and leading to the development of pulmonary hypertension (Tan et al., 2007). In broiler chickens, pulmonary hypertension, also known as ascites, is a metabolic disease characterized by the pulmonary artery hypertension-induced right ventricular hypertrophy and failure. Although evidence in human and rats indicates that dramatic changes in the function of the pulmonary vascular endothelium occur in pulmonary hypertension, little is known about these changes in broiler chicken. Our recent study demonstrated that genistein supplementation 
enhanced eNOS activity in broiler chicken (Yang et al., 2010).

To explore the mechanisms by which genistein enhanced eNOS activity, PAECs were isolated from broiler chicken embryos and treated with genistein. The rapid activation of eNOS (Figure 1) and NO release (Figure 7 ), suggested that activation of eNOS is not mediated by a genomic mechanism and this was confirmed by the treatment of cells with genistein in the absence or presence of cycloheximide or actimomycin (Figure 2 ). In consistence with this observation, genistein treatment did not affect eNOS protein expression (Figure 3). Further study showed that the rapid activation of eNOS was associated with phosphorylation of eNOS at Ser ${ }^{1179}$ (Figure 3). Although genistein has weak estrogenic effects in some tissues through binding to estrogen receptor beta with an affinity comparable with 17-estradiol, and both estrogen receptors are present in vascular cells, our data show that the rapid activation of eNOS by genistein was independent of ERs, because the specific ER antagonist ICI182, 780 did not affect genistein-induced eNOS phosphorylation and activity (Figure 4). In addition, the activation of eNOS by genistein was unrelated to tyrosine kinase inhibition (Figure 5), which is in agreement with a previous study showing that high concentration of genistein $(100 \mu \mathrm{M})$ is required to inhibits tyrosine kinase activity (Peterson and Barnes, 1996), which is much higher than the concentrations used in our study to induce maximum activation of eNOS in broiler PAECs.

Since several protein kinases, such as Akt, PKA and ERK1/2, have been proposed to phosphorylate eNOS at Ser ${ }^{1179}$ and increase eNOS activity in response to various stimuli in vascular endothelial cells (Dimmeler et al., 1999; Fulton et al., 1999; Michell et al., 1999; Bernier et al., 2000; Igarashi and Michel, 2001; Kobayashi et al., 2003), we next determine the kinses that are responsible for the activation of eNOS following genistein treatment. PKA and ERK do not appear to play a role in the genistein-induced phosphorylation of eNOS as selective kinase inhibitors failed to influence the genistein-induced eNOS activity (Figure 6) and NO release (Figure 7). In contrast, the activation of eNOS was largely abolished by PI3K or Akt inhibitors suggesting that PI3K/Akt activity contributed to the genistein-induced eNOS activation. This results are not in agreement with a recent study showing genistein induces eNOS activation through a PKA-, instead of PI3K/Akt-dependent pathway in bovine aortic endothelial cells (Liu et al., 2004). Possible explanations for this observation might include the fact we used broiler pulmonary arterial endothelial cells rather than aortic endothelial cells. Another factor might be the different dosage used in these two studies, in our study genistein concentration $(10 \mu \mathrm{M})$ is determined according to our in vivo study in broilers (Yang et al., 2010), which is ten times higher than that of Liu et al (2004). Further study using broiler aortic endothelial cells is needed to elucidate whether the discrepancy is tissue specific.

Many diverse beneficial effects of genistein on cardiovascular disease have been described in publication reports. An involvement of the endothelial vasorelaxing effects of genistein has been recently reported in human and rats (Mishra et al., 2000; Colacurci et al., 2005; Vera et al., 2007), suggesting a critical role of eNOS that contributes to the cardio-protective effect of genistein. The data presented here, along with our in vivo study (Yang et al., 2010), provided evidence that genistein activated eNOS through a non-genomic mechanism, resulting in the release of NO, restored endothelial function and attenuated pulmonary hypertension in broilers. Supplementation of genistein might be a potential therapeutic strategy that reduced the incidence of pulmonary hypertension, a leading causes of death in poultry industry.

It should be noted that in our system, PI3K or Akt inhibitor largely, but not completely abrogate genistein-induced eNOS activity, suggesting the existence of alternative signaling pathways that contributed to the phosphorylation of eNOS and related NO release. eNOS was initially reported to be phosphorylated on serine residues and has been studied in most detailed (Fleming et al., 2001). However, recent studies shown that other residues, such as threonine and tyrosine residues, can also be phosphorylated and regulates eNOS activity (Fleming et al., 1998; Harris et al., 2004). Even though the phosphorylation of eNOS at Thr495 did not change upon genistein treatment in our system (Figure 3), we can not exclude the possibility that Akt or other kinases might regulate eNOS activity through phosphorylation at other residues that attribute to the increased activity. The exact mechanism by which Akt phosphorylates eNOS in the pulmonary arterial endothelial cells is not clear. Further study is needed to address this question.

In summary, the present data demonstrated that acute genistein treatment led to activation of eNOS in broiler pulmonary arterial endothelial cells through a PI3K/Akt-dependent mechanism. Pharmacological inhibitor of Akt markedly reduced the phoshphorylation of eNOS at Ser ${ }^{1179}$ and related NO production. Our data provide an alternative 
explanation for the beneficial effect of genistein on vascular diseases in human and animals.

\section{Methods}

\section{Materials}

M199 media, FBS and other cell culture supplements were obtained from Invitrogen (Carlsbad, CA). Genistein, ICl 182, 780, inhibitors of ERK1/2 (PD98059), PKA (H89), tyrosine kinase (Herbimycin), PI3K (LY294002) and Akt (A443654) were purchased from Sigma (St. Louis, MO). Protease and phosphatase inhibitor cocktails were obtained from Roche Molecular Systems, Inc. (Alameda, CA).

\section{Cell culture}

Twenty day-old chick embryo pulmonary arterial endothelial cells (PAECs) were isolated by enzymatic dispersion according to the modified method (Visner et al., 1994). The purity of PAEC culture was characterized by indirect immunofluorescent staining for factor VIII antigen, a generally accepted marker of endothelial cells (Visner et al., 1994). Cells were cultured in M199 media supplemented with $10 \%$ FBS, $100 \mathrm{IU} / \mathrm{mL}$ penicillin, $100 \mu \mathrm{g} / \mathrm{mL}$ streptomycin, 8 $\mathrm{mM}$ HEPES, and $2 \mathrm{mM}$ glutamine at $39^{\circ} \mathrm{C}$ in a $5 \%$ $\mathrm{CO}_{2} / 95 \%$ air environment. PAECs were passaged with $0.05 \%$ trypsin and passages $4-6$ were used in all the experiments.

\section{eNOS activity}

Cells were serum-starved overnight in phenol red-free medium before eNOS activity measurements. eNOS activity was determined by measuring the conversion of $\left[{ }^{3} \mathrm{H}\right] \mathrm{L}$-arginine to $\left[{ }^{3} \mathrm{H}\right] \mathrm{L}$-citrulline as described (Hisamoto et al., 2001). In some experiments, cells were pretreated with estrogen receptor inhibitor $\mathrm{ICl} 182,780$ (100 nM); PKA inhibitor H89 $(5 \mu \mathrm{M})$, ERK inhibitor PD98059 $(10 \mu \mathrm{M})$, PI3K inhibitor LY294002 $(10 \mu \mathrm{M})$ or Akt inhibitor A443654 (5 $\mu \mathrm{M})$ for $30 \mathrm{~min}$ before incubated with genistein. The reaction was terminated by aspirating the buffer and washing with ice-cold PBS containing EGTA ( $5 \mathrm{mM})$ and EDTA (5 mM), followed by the addition of $1 \mathrm{ml}$ ice-cold trichoroacetic acid $(0.5 \mathrm{~N})$. Subsequent sample handling and then radioactivity was measured with a liquid scintillation counter. eNOS activity was normalized as picomoles of $\left[{ }^{3} \mathrm{H}\right] \mathrm{L}$ citrulline produced per milligram protein and expressed as a percentage of control.

\section{Nitric oxide measurement}

To determine the effect of genistin on NO release in vitro, serum-starved PAECs were treated with genistein in the presence or absence of kinase inhibitors, PD98059 (10 $\mu \mathrm{M})$, H89 (5 $\mu \mathrm{M})$, LY294002 (10 $\mu \mathrm{M})$, A443654 (5 $\mu \mathrm{M})$, nitric oxide was determined by measuring the sum concentration of $\mathrm{NO}_{2}^{-}$and $\mathrm{NO}_{3}{ }^{-}\left(\mathrm{NO}_{\mathrm{x}}\right)$ in culture supernatants using a fluoremetric assay kit following the manufacturer's instructions (Stratagene, La Jolla). Briefly, culture supernatants were collected and treated with $\mathrm{NO}_{3}^{-}$ for $30 \mathrm{~min}$ at room temperature to reduce $\mathrm{NO}_{3}{ }^{-}$to $\mathrm{NO}_{2}{ }^{-}$ which then reacted with 2, 3-diaminomaphthalene for 10 min to yield the fluorescent product $1(\mathrm{H})$-naphthotriazole. Fluorescence was measured with excitation and emission wavelengths of 365 and $450 \mathrm{~nm}$, respectively. Fluorescence readings were converted into concentration based on the standard curves and then represent as folds change compared to that of control.

\section{Tyrosine kinase activity assay}

Cells cultured in serum-free, phenol red-free M199 media for $24 \mathrm{~h}$ were treated with genistein as indicated in the figure legend or herbimycin $\mathrm{A}(10 \mu \mathrm{M})$ for $30 \mathrm{~min}$. Cells were harvested and lysed, and the supernatants were used for the tyrosine kinase activity assay according to method described (Liu et al., 2004). The relative fluorescence intensity were normalized to corresponding protein levels and expressed as percentage of the controls.

\section{Western blotting}

Cells were lysed in ice-cold buffer containing $20 \mathrm{mM}$ Tris-HCl (pH 7.4), $2.5 \mathrm{mM}$ EDTA, 1\% Triton X-100, 1\% sodium deoxycholate, $0.1 \%$ SDS, $50 \mathrm{mM} \mathrm{NaF}, 1 \mathrm{mM}$ $\mathrm{Na}_{3} \mathrm{VO}_{4}$ and $1 \mathrm{mM}$ protease inhibitor cocktail from Roche (Alameda, CA). Cell lysates were centrifuged for $15 \mathrm{~min}$ at $12,000 \mathrm{~g}$ to remove cellular debris. Equal amounts of protein were separated on SDS-page gels and transfer to PVDF membranes (Millpore, Bedford, MA.). The membranes were blocked in a $5 \%$ skimmed milk solution at room temperature for $1 \mathrm{~h}$, and then incubated with antiphospho-eNOS Ser ${ }^{1179}$, anti-phospho-eNOS $\operatorname{Thr}^{497}$ from upstate (Upstate Biotechnologies, Lake Placid, NY), or anti-eNOS antibody from Transduction Laboratories (Lexington, $\mathrm{KY}$ ). Blots were stripped and re-probed with anti-actin antibody (Sigma) to demonstrated equal loading.

\section{Statistical analysis}

Comparisons between groups were performed using oneway ANOVA followed by the Duncan test. Differences were considered statistically significant at the level of $P<0.05$ and values are represented as means \pm S.D. The statistical analysis was performed with the software of SPSS 11.0 for Windows.

\section{Acknowledgements}

This work was supported by Yangtz River Scholar and Innovation Research Team Development Program (Project No. IRT0945), and by the grants from the National Natural Science Foundation of China (NO. 30700576) and State Key Laboratory of Animal Nutrition (Project No. 2004DA125184-0807).

\section{References}

Barrett-Connor E, Bush TL. Estrogen and coronary heart disease in women. JAMA 1991;265:1861-7 
Bernier SG, Haldar S, Michel T. Bradykinin-regulated interactions of the mitogen-activated protein kinase pathway with the endothelial nitric-oxide synthase. J Biol Chem 2000;275:30707-15

Boo YC, Sorescu G, Boyd N, Shiojima I, Walsh K, Du J, Jo $\mathrm{H}$. Shear stress stimulates phosphorylation of endothelial nitric-oxide synthase at Ser1179 by Akt-independent mechanisms: role of protein kinase A. J Biol Chem 2002; 277:3388-96

Cassidy A, Griffin B. Phyto-oestrogens:a potential role in the prevention of CHD? Proc Nutr Soc 1999;58:193-9

Catania MA, Crupi A, Firenzuoli F, Parisi A, Sturiale A, Squadrito F, Caputi AP, Calapai G. Oral administration of a soy extract improves endothelial dysfunction in ovariectomized rats. Planta Med 2002;68:1142-4

Chen Z, Yuhanna IS, Galcheva-Gargova Z, Karas RH, Mendelsohn ME, Shaul PW. Estrogen receptor alpha mediates the nongenomic activation of endothelial nitric oxide synthase by estrogen. J Clin Invest 1999;103:401-6

Colacurci N, Chiantera A, Fornaro F, de Novellis V, Manzella D, Arciello A, Chiantera V, Improta L, Paolisso G. Effects of soy isoflavones on endothelial function in healthy postmenopausal women. Menopause 2005;12:299-307

Cuevas AM, Irribarra VL, Castillo OA, Yanez MD, Germain AM. Isolated soy protein improves endothelial function in postmenopausal hypercholesterolemic women. Eur J Clin Nutr 2003;57:889-94

Dimmeler S, Fleming I, Fisslthaler B, Hermann C, Busse R, Zeiher AM. Activation of nitric oxide synthase in endothelial cells by Akt-dependent phosphorylation. Nature 1999; 399:601-5

Farhat MY, Lavigne MC, Ramwell PW. The vascular protective effects of estrogen. FASEB J 1996;10:615-24

Fleming I, Bauersachs J, Fisslthaler B, Busse R. Ca2+-independent activation of the endothelial nitric oxide synthase in response to tyrosine phosphatase inhibitors and fluid shear stress. Circ Res 1998;82:686-95

Fleming I, Fisslthaler B, Dimmeler S, Kemp BE, Busse R. Phosphorylation of $\mathrm{Thr}(495)$ regulates $\mathrm{Ca}(2+)$ /calmodulindependent endothelial nitric oxide synthase activity. Circ Res 2001;88:E68-75

Fulton D, Gratton JP, McCabe TJ, Fontana J, Fujio Y, Walsh K, Franke TF, Papapetropoulos A, Sessa WC. Regulation of endothelium-derived nitric oxide production by the protein kinase Akt. Nature 1999;399:597-601

Grodstein F, Stampfer MJ, Manson JE, Colditz GA, Willett WC, Rosner B, Speizer FE, Hennekens CH. Postmenopausal estrogen and progestin use and the risk of cardiovascular disease. N Engl J Med 1996;335:453-61

Harris MB, Blackstone MA, Sood SG, Li C, Goolsby JM, Venema VJ, Kemp BE, Venema RC. Acute activation and phosphorylation of endothelial nitric oxide synthase by HMG-CoA reductase inhibitors. Am J Physiol Heart Circ Physiol 2004;287:H560-6

Hisamoto K, Ohmichi M, Kurachi H, Hayakawa J, Kanda Y, Nishio Y, Adachi K, Tasaka K, Miyoshi E, Fujiwara N,
Taniguchi N, Murata Y. Estrogen induces the Akt-dependent activation of endothelial nitric-oxide synthase in vascular endothelial cells. J Biol Chem 2001;276:3459-67

Igarashi J, Michel T. Sphingosine 1-phosphate and isoform-specific activation of phosphoinositide 3-kinase beta. Evidence for divergence and convergence of receptorregulated endothelial nitric-oxide synthase signaling pathways. J Biol Chem 2001;276:36281-8

Karamsetty MR, Klinger JR, Hill NS. Phytoestrogens restore nitric oxide-mediated relaxation in isolated pulmonary arteries from chronically hypoxic rats. J Pharmacol Exp Ther 2001;297:968-74

Kobayashi N, Mita S, Yoshida K, Honda T, Kobayashi T, Hara K, Nakano S, Tsubokou Y, Matsuoka H. Celiprolol activates eNOS through the PI3K-Akt pathway and inhibits VCAM-1 Via NF-kappaB induced by oxidative stress. Hypertension 2003;42:1004-13

Kuiper GG, Carlsson B, Grandien K, Enmark E, Haggblad J, Nilsson S, Gustafsson JA. Comparison of the ligand binding specificity and transcript tissue distribution of estrogen receptors alpha and beta. Endocrinology 1997;138:863-70

Lavallee M, Takamura M, Parent R, Thorin E. Crosstalk between endothelin and nitric oxide in the control of vascular tone. Heart Fail Rev 2001;6:265-76

$\mathrm{Li} \mathrm{H}$, Forstermann $\mathrm{U}$. Nitric oxide in the pathogenesis of vascular disease. J Pathol 2000;190:244-54

Lissin LW, Cooke JP. Phytoestrogens and cardiovascular health. J Am Coll Cardiol 2000;35:1403-10

Liu D, Homan LL, Dillon JS. Genistein acutely stimulates nitric oxide synthesis in vascular endothelial cells by a cyclic adenosine $5^{\prime}$-monophosphate-dependent mechanism. Endocrinology 2004;145:5532-9

MacRitchie AN, Jun SS, Chen Z, German Z, Yuhanna IS, Sherman TS, Shaul PW. Estrogen upregulates endothelial nitric oxide synthase gene expression in fetal pulmonary artery endothelium. Circ Res 1997;81:355-62

Mawji IA, Marsden PA. Perturbations in paracrine control of the circulation:role of the endothelial-derived vasomediators, endothelin-1 and nitric oxide. Microsc Res Tech 2003;60:46-58

Michell BJ, Griffiths JE, Mitchelhill KI, Rodriguez-Crespo I, Tiganis T, Bozinovski S, de Montellano PR, Kemp BE, Pearson RB. The Akt kinase signals directly to endothelial nitric oxide synthase. Curr Biol 1999;9:845-8

Mishra SK, Abbot SE, Choudhury Z, Cheng M, Khatab N, Maycock NJ, Zavery A, Aaronson PI. Endothelium-dependent relaxation of rat aorta and main pulmonary artery by the phytoestrogens genistein and daidzein. Cardiovasc Res 2000;46:539-46

Peterson G, Barnes S. Genistein inhibits both estrogen and growth factor-stimulated proliferation of human breast cancer cells. Cell Growth Differ 1996;7:1345-51

Schiffrin EL. A critical review of the role of endothelial factors in the pathogenesis of hypertension. J Cardiovasc Pharmacol 2001;38 Suppl 2:S3-6 
Squadrito F, Altavilla D, Squadrito G, Saitta A, Cucinotta D, Minutoli L, Deodato B, Ferlito M, Campo GM, Bova A, Caputi AP. Genistein supplementation and estrogen replacement therapy improve endothelial dysfunction induced by ovariectomy in rats. Cardiovasc Res 2000;45:454-62

Stampfer MJ, Colditz GA. Estrogen replacement therapy and coronary heart disease:a quantitative assessment of the epidemiologic evidence. Prev Med 1991;20:47-63

Tan X, Hu SH, Wang XL. Possible role of nitric oxide in the pathogenesis of pulmonary hypertension in broilers:a synopsis. Avian Pathol 2007;36:261-7

Vera R, Sanchez M, Galisteo M, Villar IC, Jimenez R, Zarzuelo A, Perez-Vizcaino F, Duarte J. Chronic admi- nistration of genistein improves endothelial dysfunction in spontaneously hypertensive rats:involvement of eNOS caveolin and calmodulin expression and NADPH oxidase activity. Clin Sci (Lond) 2007;112:183-91

Visner GA, Staples ED, Chesrown SE, Block ER, Zander DS, Nick HS. Isolation and maintenance of human pulmonary artery endothelial cells in culture isolated from transplant donors. Am J Physiol 1994;267:L406-13

Yang Y, Gao M, Wu Z, Guo Y. Genistein attenuates low temperature induced-pulmonary hypertension in broiler chicks by modulating endothelial function. Eur $\mathrm{J}$ Pharmacol 2010;649:242-8 